\section{ESR Method for the Detection of Irradiated Unboned Meats and Seafood}

\author{
Makoto Miyahara, ${ }^{*}, a$ Takeo Nagasawa, ${ }^{b}$ \\ Shigeru Akiyama, ${ }^{b}$ Yasuhiko Kobayashi, ${ }^{c}$ \\ Toshiki Mashimizu, ${ }^{d}$ and Tamio Maitani ${ }^{a}$
}

${ }^{a}$ National Institute of Health Sciences, 1-18-1 Kamiyoga, Setagaya-ward, Tokyo, 158-8501, Japan, ${ }^{b}$ School of Allied Health Sciences, Kitasato University, 1-15-1 Kitasato Sagamihara City, Kanagawa 228-8555, Japan, 'Japan Atomic Energy Research Institute, 1233 Watanuki-cho, Takasaki, 3701207, Japan, and 'Japan Electronic Optics Laboratory, 1156 Nakagami-machi, Akishima, Tokyo, 196-0022, Japan

(Received April 27, 2004; Accepted June 2, 2004; Published online June 2, 2004)

ESR methods for detecting irradiated foods are used internationally. In the present study, an identification method for irradiated unboned meats, fish with bone, and shellfish were studied. These foods were found to give ESR signals that were specific for irradiated samples. The signals appeared to originate from hydroxyapatite, calcium carbonate, collagen, chitin, melanin, and/or other complex materials. The shape of the ESR spectra for irradiated samples can be complex and difficult to analyze. Each ESR spectrum in the present study had several peaks, some of which were not affected by the doses applied. Some peak intensities, however, did increase with increases in dose. Minimum detectable dose was approximately 1 to $0.5 \mathrm{kGy}$. These results indicate that the shape of the ESR spectrum depends on the sample components.

Key words — irradiated foods, ESR method, meat with bone, shellfish

\section{INTRODUCTION}

Food irradiation is a technology for sterilizing foods contaminated by bacteria and is expected to reduce the incidence of food-borne illness. Irradiated foods are required to be labeling that they were treated with an ionization beam. Standard ESR methods for detection of irradiated foods have been ap-

\footnotetext{
*To whom correspondence should be addressed: National Institute of Health Sciences, 1-18-1 Kamiyoga Setgaya, Tokyo 1588501, Japan. Tel.:+81-3-3700-1141; Fax: +81-3-3707-6950; Email:mmiyaha@nihs.go.jp
}

proved for international use. ${ }^{1-3)}$ These methods are used for several groups of irradiated foods, but there has been interest in applying the methods to additional food groups. ESR spectroscopy has a number of strong points, including simple sample pretreatment and easy operation of ESR machine. It also, however, has weak points: the ESR signal intensity is affected by the water content of the sample and the sample shape; the shape of the ESR spectrum can change in response to heavy metal impurities or by the presence of organic radicals in the sample; most ESR radicals can fade out during storage. To compensate for these weaknesses, we are developping a new reproducible procedure for sample preparation. ${ }^{4)}$ Based on this procedure, a new approach to the detection of irradiated foods will be reported herein.

\section{MATERIALS AND METHODS}

Irradiation Apparatus —— A wet type ${ }^{60} \mathrm{Co}$ plate source was used at the Japan Atomic Energy Research Institute. Absorbed doses were measured with a Gammachrome YR. AEA (Oxon, U.K.).

Irradiation Process — Five grams of each sample were placed in a 10-ml Pyrex tube. Samples were irradiated at $10^{\circ} \mathrm{C}$. After irradiation, the samples were stored at $-20^{\circ} \mathrm{C}$.

Sample — Flesh samples were purchased at a local supermarket in the Setagaya area.

ESR Spectrometer — Jeol model FA200 RE, and FX. Operating conditions: frequency, $9.432 \mathrm{GHz}$; scan field, $333.5 \mathrm{mT}$; scan width, $7.5 \mathrm{mT}$; modulation frequency, $100 \mathrm{kHz}$; modulation width, $0.32 \mathrm{mT}$; power, $1 \mathrm{~mW}$.

Sample Preparation — Bones were separated from the meat and washed with water. Small pieces of meat attached to the bones were carefully removed. The bones were dried over $\mathrm{P}_{2} \mathrm{O}_{5}$ under vacuum for $6 \mathrm{hr}$. Shells of the shellfish were dried in the same manner described above.

ESR Sample Preparation — Dried samples were crushed with a hammer or garlic crusher. The small pieces of bone were screened with sieves. The sample diameters ranged from 1-2 $\mathrm{mm}$. The screened bones were packed in an ESR sample tube (4 mm i.d.).

Determination of the Spin Density — An external standard method was used for detection of the spin contents in the sample. Mn marker signals were used for reference for the g-value. 

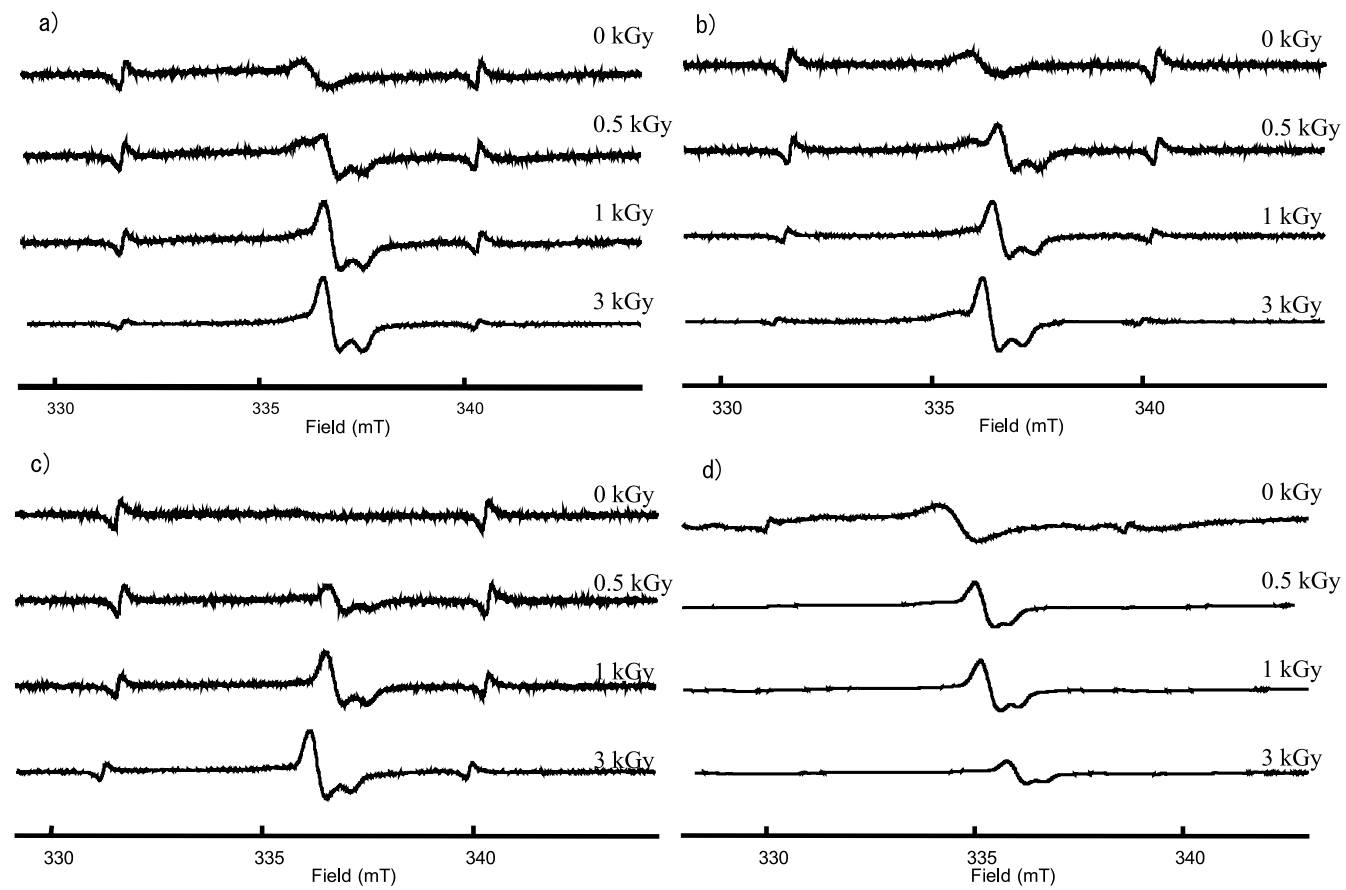

Fig. 1. ESR Spectra of Irradiated Bones in Frog Leg (a), Wing-Tip of Chicken (b), in Fish (c) and Shells of Clams (d) at 0, 0.5, 1, and 3 kGy

\section{RESULTS AND DISCUSSION}

\section{ESR Spectra}

Samples (frog leg bone, wing-tip bone of chicken, rib of black rockfish, and shells of clams) were irradiated at several doses at $10^{\circ} \mathrm{C}$. The results are shown in Fig. 1. ESR Spectra of nonirradiated bones of frog legs, bones of wing tips of chicken, and shell of clams showed broad peaks. But ESR spectrum of nonirradiated ribs of black rockfish did not. Those signals have been explained that they originated from collagen in those samples. The baseline of ESR spectrum for shell of clams was rolling and suggested intercalations of heavy metals in the hydroxyapatite crystal structures. Those ESR spectra of irradiated samples gave three peaks as shown in Fig. 1. g-Value of the most intense peak in each spectrum was same and was 2.002. This result means those signals were originated from common chemical species. The chemical components of hydroxyapatite are similar to each other although they are belonging to completely different biological species. From those spectra, minimum detectable doses for frog leg bone, wing-tip bone of chicken, rib of black rockfish, and shell of clams were $1,1,1$, and $0.5 \mathrm{kGy}$, respectively.

ESR spectra of nonirradiated and irradiated shrimps are shown in Fig. 2. These charts show a single broad peak and are different from those in

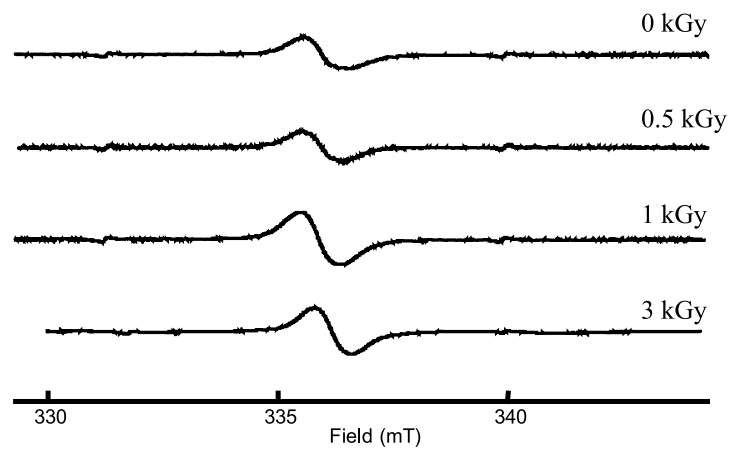

Fig. 2. ESR Spectra of Irradiated Shrimp Shells at 0, 0.5, 1, and $3 \mathrm{kGy}$

Fig. 1. This peak may originate from the chitin in the shell. Some spectra showed signals from manganese ions (data not shown).

\section{Dose Responses of ESR Signal Intensities}

The signal intensities of three ESR peaks vs. the absorbed dose are shown in Fig. 3. The most sensitive peak of the ESR signal for irradiated frog leg bone had a g-value of 2.002. This peak intensity was not saturated in the dose range we examined. However, those of the other samples (wing-tip bone of chicken, rib of black rockfish, and shell of clams) were not linear with respect to dose and were not saturated in the high-dose region. The peak intensities at $g=1.997$ in ESR spectra of frog leg bone, 

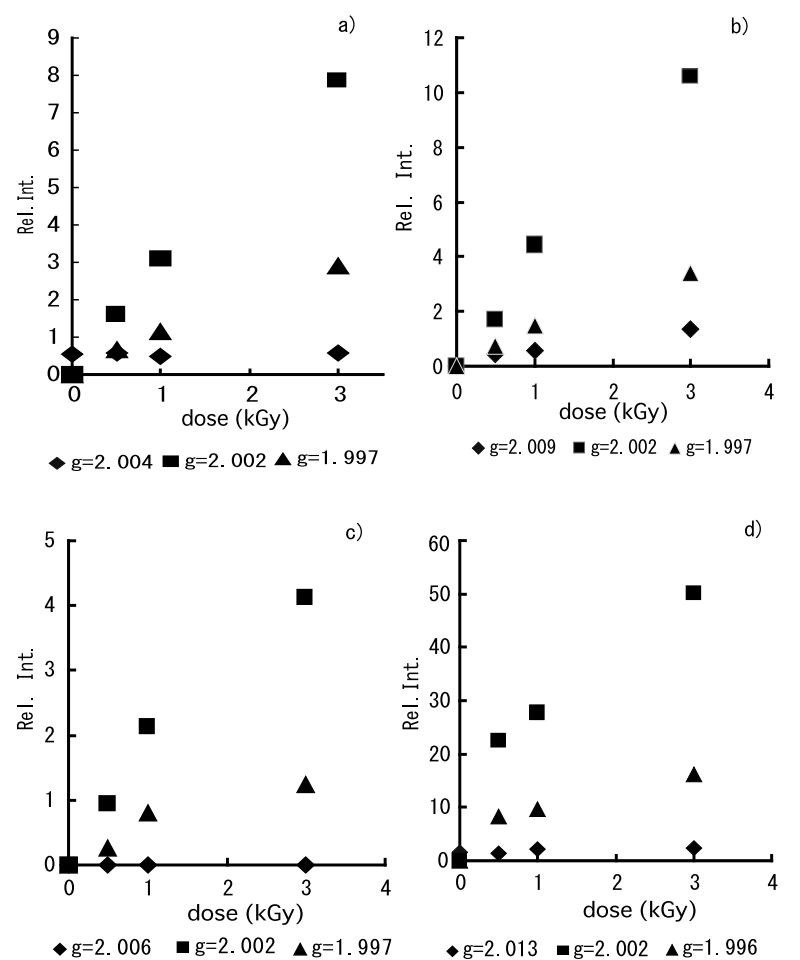

Fig. 3. Dose-Response Curves for ESR Signal Intensity

(a) Frog leg bone, (b) wing-tip bone of chicken, (c) rib of black rock fish and (d) shells of clams. All data indicated are means of three measurements (S.D. $<0.01$ )

wing-tip bone of chicken, rib of black rockfish, and shell of clams were not linear with respect to dose, as these peaks overlapped with peaks at $g=2.002$. The intensities of the peaks at 2.004 in the ESR spectra of frog leg bone and at 2.0057 of black rockfish rib did not change as the dose increased. In contrast, the corresponding peaks of wing-tip bone of chicken $(g=2.0087)$ and shell of clams $(g=2.0125)$ were slightly increased as the dose increased. Thus, the spectra of these samples were quite similar to each other, although the responses to irradiation were quite different.

Figure 4 shows the dose response curve of an irradiated shrimp shell.

The intensity was not linear with respect to dose. As a result, it was difficult to estimate the absorbed dose from the curve.

The ESR method can successfully identify irradiated frog leg bone, wing-tip bone of chicken, rib of black rockfish, and shell of clams. The absorbed dose of some samples can be estimated by doseresponse curves. However, the ESR spectrum of an irradiated sample can vary from sample to sample. Careful use of this method is recommended.

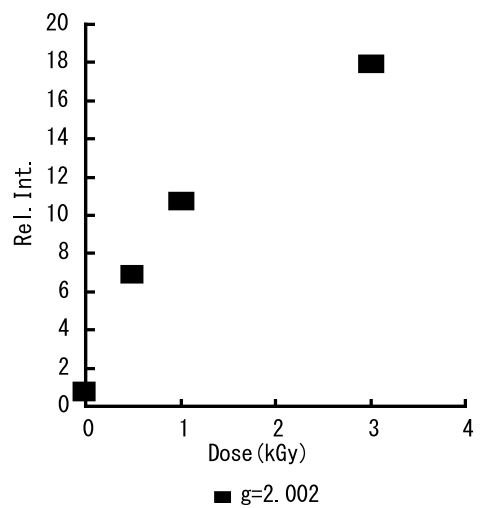

Fig. 4. Dose-Response Curves for ESR Signal Intensity of Irradiated Shrimp Shells

Acknowledgements This study was financially supported by the Budget for Nuclear Research of the Ministry of Education, Culture, Sports, Science, and Technology of Japan, based on screening and counseling by the Atomic Energy Commission.

We would like to thank Dr. K. Morehouse and Dr. C. Warner of the US Food and Drug Administration, Washington, DC, and Dr. M. F. Desrosiers, US National Institute of Standards and Technology, for their useful advice and discussion.

\section{REFERENCES}

1) Codex Alimentarius Commission (2002) Proposed draft recommended international code of practice for radiation processing of food. In Report of the 34th session of the Codex Committee on Food Additives and Contaminants, Joint office for FAO/ WHO, Rome, ALINORM 03/12 Appendix V, pp. 73-79.

2) Codex Alimentarius Commission (2001) Proposed draft revised codex general standard for irradiated foods (at step 5 of the procedure). In Report of the 33rd Session of the Codex Committee on Food Additives and Contaminants, Joint office for FAO/ WHO, Rome, ALINORM 01/12A, Appendix VII, pp. 267-269.

3) Codex Alimentarius Commission (2001) Proposed draft revised codex general standard for irradiated foods (at step 5 of the procedure). In Report of the 33rd Session of the Codex Committee on Food Additives and Contaminants, Joint office for FAO/ WHO, Rome, ALINORM 01/41, para.197-200, p. 27.

4) Miyahara, M., Nagasawa, T., Kamimura, T., Ito, H., Toyoda, M. and Saito, Y. (2002) Identification of irradiation of boned chicken by determination of otyrosine and electron spin resonance spectrometry. J. Health Sci., 48, 79-82. 